\title{
Synthesis and Antimicrobial Activity of New Thiazolidinone Derivatives With the use of $\boldsymbol{y}$-Ferrite Catalyst
}

\author{
S. P. SHRIVASTAVA, NARESHVARMA SEELAM ${ }^{*}$ and RACHANA RAI
}

Heterocyclic Research laboratory, Department of Chemistry

Dr. H. S. Gour University, Sagar, M. P, 470003-India

utd_naresh@yahoo.co.in

Received 9 August 2011; Accepted 4 October 2011

\begin{abstract}
A series of compounds were synthesized from 3,3'-(pyridine-2,6diyl) bis (2-phenylthiazolidin-4-one(1), which was prepared from a one-pot three component condensation reaction of 2, 6-diamino pyridine, benzaldehyde and thioglycolic acid in the presence of $\gamma$-ferrite as a catalyst. The newly synthesized compounds were characterized by IR, ${ }^{1} \mathrm{HNMR},{ }^{13} \mathrm{C}$ NMR spectral data. All compounds were tested for anti bacterial and anti fungal activities.
\end{abstract}

Keywords: Pyridine, Azo derivatives, $\gamma$-Ferrite, Arylidene derivatives.

\section{Introduction}

During the past decade, combinatorial chemistry has provided access to chemical libraries based on privileged structures ${ }^{1}$, with heterocyclic structures receiving special attention as they belong to a class of compounds with proven utility in medicinal chemistry ${ }^{2}$. There has been considerable interest in the chemistry of thiazolidin-4-one ring system, which is a core structure in various synthetic pharmaceuticals displaying a broad spectrum of biological activities $^{3}$. Thiazolidinones and their derivatives are an important group of heterocyclic compounds, having valuable biological activities in the areas of medicine and agriculture ${ }^{4}$. Thiazolidinone ring occurs in nature; thus actithiazic acid[(-)2-(5-carboxypentyl)thiazolidin4-one)] isolated from streptomyces strains exhibits highly specific in vitro activity against mycobacterium tuberculosis ${ }^{5}$. They have found uses, for example, as insecticides, tuberculostatic ${ }^{6-7}$, anti-inflammatory ${ }^{8}, \mathrm{CNS}$ stimulant, anthelmintic ${ }^{9}, \mathrm{CO}^{2+}$ channel blocker ${ }^{10}$, antiviral $^{11}$, analgesic ${ }^{12}$, anticonvulsant ${ }^{13}$, anti platelet activating factor ${ }^{14}$, anticancer ${ }^{15}$, and anti HIV $^{16}$. 4-Thiazolidinones have also been reported as novel inhibitors of bacterial enzyme $^{17}$. In view of these above findings it was thought of interest to accommodate thiazolidin-4-one and pyridine in a single molecular frame work and screened for their anti microbial activity. 


\section{Experimental}

The NMR $\left({ }^{1} \mathrm{H} \&{ }^{13} \mathrm{C}\right)$ spectra were recorded at $400 \mathrm{MHz}, 100 \mathrm{MHz}$ respectively with a Bruker Avance II 400 instrument. NMR spectra were obtained in solutions of DMSO (d6) and chemical shifts reported in parts per million (ppm) and TMS as an internal standard. Melting points were determined in an open capillary and are uncorrected. IR spectra were recorder on a FT-IR Shimadzu 8400 Spectrophotometer and reported in wave numbers $\left(\mathrm{cm}^{-1}\right)$.

\section{3,3'-(Pyridine-2,6-diyl)bis(2-phenylthiazolidin-4-one(1)}

To a mixture of 2,6-di amino pyridine(1 equiv.), benzaldehyde (2 equiv), anhydrous $\gamma$-ferrite $\left(\mathrm{Fe}_{2} \mathrm{O}_{3} ; 4\right.$ equiv) was added and the reaction mixture was refluxed with constant stirring in dry benzene ( $40 \mathrm{ml}$ ) for $1 / 2 \mathrm{~h}$, followed by the addition of thioglycolic acid ( 2 equiv). The refluxing and stirring were continued for another $10 \mathrm{~h}$. The reaction was monitored by TLC. After the completion of the reaction, a reddish brown amorphous solid $\mathrm{Fe}_{2} \mathrm{O}_{3} \cdot 2 \mathrm{H}_{2} \mathrm{O} / \mathrm{FeO}(\mathrm{OH})$ was removed by filtration, filtrate was concentrated to dryness under reduced pressure to achieve compound.

Dark brown crystals; yield 74\%, m.p: $256^{\circ}-259^{\circ} \mathrm{c}$; IR $\left(\mathrm{KBr}, v \max , \mathrm{cm}^{-1}\right) 1698(\mathrm{C}=0)$, 1130(C-N), 672(C-S), $1612(\mathrm{C}=\mathrm{N}), 1597(\mathrm{C}-\mathrm{C}),{ }^{1} \mathrm{H}$ NMR ( $\delta$ ppm, DMSO-d6) $3.71(\mathrm{~s}, 4 \mathrm{H}$, $\left.\mathrm{S}-\mathrm{CH}_{2}\right), 5.18(\mathrm{~s}, 2 \mathrm{H} \mathrm{N}-\mathrm{CH}), 7.32(\mathrm{~m}, 10 \mathrm{H}, \mathrm{Ar}-\mathrm{H}), 7.89\left(\mathrm{t}, 1 \mathrm{H}\right.$, pyridine $\left.\mathrm{C}_{4}\right), 8.2(\mathrm{~d}, 2 \mathrm{H}$, pyridine $\mathrm{C}_{3}, \mathrm{C}_{5}$ ) ${ }^{13} \mathrm{C}-\mathrm{NMR}\left(\mathrm{DMSO}, 100 \mathrm{MH}_{\mathrm{z}}\right.$ ): 172.1, 152, 139.74, 131.04, 127.4, 38.7 .

General procedure for the Synthesis of 3,3'-(pyridine-2,6-diyl)bis(5-substitutedarylidene-2-phenyl) thiazolidin-4-one derivatives (2a-j)

A mixture of compound 1 (1 equiv.), aromatic aldehyde ( 2 equiv.), anhydrous sodium acetate (2equiv.) in glacial acetic acid (50ml) was heated under reflux for $4 \mathrm{~h}$. Concentrated, cooled and poured into the crushed ice. The solid thus separated was filtered, washed with water and recrystallized. All the compounds $\mathbf{2 a - j}$ were prepared by the same methodology as mentioned above. The physical and analytical data of compounds were mentioned in Table 1 .

General procedure for the synthesis of 3,3'-(pyridine-2,6-diyl)bis(5-(phenyl substituted diazenyl)-2-phenyl)thiazolidin-4-one 3(a-c)

To a solution of aniline $(0.01 \mathrm{~mol})$ in glacial acetic acid $(5 \mathrm{~mL})$ was added conc. $\mathrm{HCl}(3 \mathrm{~mL})$ at $0-5^{\circ} \mathrm{C}$. A Solution of Sodium nitrite $(1 \mathrm{~g}$ in $5 \mathrm{~mL}$ of water) was then added drop wise. The diazonium salt Solution thus prepared was added to a solution of compound"1" $(0.01 \mathrm{~mol})$ in DMF drop wise with stirring below $0{ }^{\circ} \mathrm{C}$. The reaction mixture were kept at room temperature for 2-3 days and then poured in to cold water $(200 \mathrm{~mL})$. The resulting solids were washed with water and recrystallized from suitable solvents. (viz, Ethanol, methanol). The physical \& analytical data of all synthesized compounds were given in Table 1 .

\section{3,3'-(Pyridine-2,6-diyl)bis(5-(4-hydroxy arylidene-2-phenyl)thiazolidin-4-one (2a)}

IR $\left(\mathrm{KBr}, \mathrm{cm}^{-1}\right)$ vmax $=3182.65(\mathrm{O}-\mathrm{H}), 3120.93(\mathrm{CH}$ in $\mathrm{ring}), 3057.27(\mathrm{Ar}), 1653.05(\mathrm{C}=\mathrm{O})$, $1415.80(\mathrm{C}-\mathrm{N}), 1600,1625(\mathrm{C}=\mathrm{C}$ and $\mathrm{C}=\mathrm{N}) ;{ }^{1} \mathrm{H}$ NMR $\left(\delta \mathrm{ppm}, \mathrm{DMSO}-\mathrm{d}_{6}\right), 6.25(\mathrm{~s}, 2 \mathrm{H}$, $\mathrm{N}-\mathrm{CH}), 6.9$ (t, 1H, Pyridine C4), $7.35(\mathrm{~s}, 2 \mathrm{H}, \mathrm{CH}), 7.6(\mathrm{~m}, 18 \mathrm{H}, \mathrm{Ar}-\mathrm{H}), 8.0(\mathrm{~s}, 2 \mathrm{H}, \mathrm{OH}), 8.21$ (d, $2 \mathrm{H}, \mathrm{C}_{2}-\mathrm{H}$ and $\mathrm{C}_{5}-\mathrm{H}$ in pyridine); ${ }^{13} \mathrm{C}$ NMR (DMSO) $\delta: 170.8,155.08,137.02,135.8$, $132.5,122.91,118.2,114.86$. 


\section{3,3'-(Pyridine-2,6-diyl)bis(5-(arylidene-2-phenyl)thiazolidin-4-one (2b)}

IR(KBr, cm $\left.{ }^{-1}\right) v \max =3049($ Ar-C-H $), 1704(\mathrm{C}=0), 1625(\mathrm{C}=\mathrm{C}), 1620(\mathrm{C}=\mathrm{N}), 1515(\mathrm{C}-\mathrm{N})$, ${ }^{1} \mathrm{H}$ NMR ( $\delta$ ppm, DMSO-d6), 8.71(m, 20H, Ar-H), 6.20 (s, 2H,N-CH-Ar), 6.92 (t, 1H, C 4 - H in Pyridine), 8.19 (d, 2H, $\mathrm{C}_{3}-\mathrm{H}, \mathrm{C}_{5}-\mathrm{H}$ in pyridine); ${ }^{13} \mathrm{C}$ NMR (DMSO) $\delta: 170.79,156.2$, $136.1,129.8,109.12$.

3,3'-(Pyridine-2,6-diyl)bis(5-(4-N-dimethylaminoarylidene-2-phenyl)thiazolidin-4one (2c)

IR $\left(\mathrm{KBr}, \mathrm{cm}^{-1}\right) v \max =3423.76(\mathrm{~N}-\mathrm{H}), 3080.42(\mathrm{Ar}), 3030.27(\mathrm{CH}$ in alkene $), 2931.90\left(\mathrm{CH}_{3}\right)$, 1683.91(C=O), 1597.11(C-C), $1417.73(\mathrm{C}-\mathrm{N}), 1361.79\left(3^{\circ}\right.$ amine).

3,3'-(Pyridine-2,6-diyl)bis(5-(3,4,5-tri methoxy arylidene-2-phenyl)thiazolidin-4-one (2d) $\operatorname{IR}\left(\mathrm{KBr}, \mathrm{cm}^{-1}\right)$ vmax $=3080.42(\mathrm{Ar}), 3057.27\left(\mathrm{C}-\mathrm{H}\right.$ in ring), 2937.68 $\left(\mathrm{CH}_{3}\right), 1624.12(\mathrm{C}=\mathrm{O})$, 1388.79(C-N), 1230.63 (C-O-C), 702.11(C-S); ${ }^{1} \mathrm{H}$ NMR ( $\delta$ ppm, DMS0-d6) 3.86(s, 12H, 3,5- $\left.\mathrm{OCH}_{3}\right) 3.79\left(\mathrm{~s}, 6 \mathrm{H}, 4-\mathrm{OCH}_{3}\right) 7.15-7.25(\mathrm{~m}, 14 \mathrm{H}, \mathrm{Ar}-\mathrm{H}), 7.5(\mathrm{~d}, 2 \mathrm{H}, 3,5$-Pyridine), 7.96 (s, $2 \mathrm{H}, \mathrm{CH}), 8.07(\mathrm{~s}, 2 \mathrm{H}, \mathrm{C}-\mathrm{H}), 8.30\left(\mathrm{t}, 1 \mathrm{H}, \mathrm{C}_{4}-\mathrm{H}\right.$ in pyridine); ${ }^{13} \mathrm{C}$ NMR (DMSO) $\delta: 171.3$, $158.9,153.89,137.2,131.9,124.01,118.7,114.2,56.2$.

3,3'-(Pyridine-2,6-diyl)bis(5-(4-methoxy arylidene-2-phenyl)thiazolidin-4-one (2e) IR $\left(\mathrm{KBr}, \mathrm{cm}^{-1}\right)$ vmax $=3057.27(\mathrm{Ar}), 3030.27\left(\mathrm{CH}\right.$ in ring), 2933.83( $\left.\mathrm{CH}_{3}\right), 1622.19(\mathrm{C}=\mathrm{O}), 1394.58$ (C-N), 1219.05(C-O-C), 640.39 (C-S). ${ }^{1} \mathrm{H}$ NMR ( $8 \mathrm{ppm}$, DMSO-d6) $3.73\left(\mathrm{~s}, 6 \mathrm{H}, \mathrm{OCH}_{3}\right), 7.35$ $(\mathrm{m}, 18 \mathrm{H}, \mathrm{Ar}-\mathrm{H}), 7.67\left(\mathrm{t}, 1 \mathrm{H}, \mathrm{C}_{4}-\mathrm{H}\right.$ in pyridine), $7.9\left(\mathrm{~d}, 2 \mathrm{H}, \mathrm{C}_{3}-\mathrm{H}\right.$ and $\mathrm{C}_{5}-\mathrm{H}$ in pyridine), 8.09 (s, $\left.2 \mathrm{H}, \mathrm{C}-\mathrm{H}\right)$;

\section{3,3'-(Pyridine-2,6-diyl)bis(5-(2-chloro arylidene-2-phenyl)thiazolidin-4-one (2f)}

$\mathrm{IR}\left(\mathrm{KBr}, \mathrm{cm}^{-1}\right) \quad v \max =3082.35(\mathrm{Ar}), 3059.20(\mathrm{C}-\mathrm{H}$ in heterocyclic ring $), 1653.05(\mathrm{C}=\mathrm{O})$, $1388.79(\mathrm{C}-\mathrm{N}), 1089.82(\mathrm{C}-\mathrm{Cl}), 702.11(\mathrm{C}-\mathrm{S})$.

\section{3,3'-(Pyridine-2,6-diyl)bis(5-(2-bromo arylidene-2-phenyl)thiazolidin-4-one (2g)}

$\mathrm{IR}\left(\mathrm{KBr}, \mathrm{cm}^{-1}\right)$ vmax=3058.91(C-H in heterocyclic ring), $3027.18(\mathrm{Ar}), 1638.07(\mathrm{C}=\mathrm{O})$, $1387.12(\mathrm{C}-\mathrm{N}), 686.17(\mathrm{C}-\mathrm{S})$.

3,3'-(Pyridine-2,6-diyl)bis(5-(4-nitro arylidene-2-phenyl)thiazolidin-4-one (2h)

$\mathrm{IR}\left(\mathrm{KBr}, \mathrm{cm}^{-1}\right)$ vmax $=3057.93(\mathrm{CH}$ in ring), $3018(\mathrm{Ar}-\mathrm{CH}), 1637.11(\mathrm{C}=\mathrm{O}), 1530.98(\mathrm{~N}=\mathrm{O})$, 1387.54(C-N), 701.08(C-S); ${ }^{1} \mathrm{H}$ NMR ( $\delta$ ppm, DMSO-d6) 5.91(s, 2H, CH), 7.1-7.4(m,18H,Ar$\mathrm{H}), 7.89\left(\mathrm{t}, 1 \mathrm{H}, \mathrm{C}_{4}-\mathrm{H}\right.$ in pyridine), $8.02(\mathrm{~s}, 2 \mathrm{H}, \mathrm{CH}), 8.35(\mathrm{~d}, 2 \mathrm{H}, 3,5-\mathrm{H}$ in pyridine).

\section{3,3'-(Pyridine-2,6-diyl)bis(5-(4-chloro arylidene-2-phenyl)thiazolidin-4-one (2i):}

$\mathrm{IR}\left(\mathrm{KBr}, \mathrm{cm}^{-1}\right) v \max =3082.35(\mathrm{Ar}), 3059.20(\mathrm{C}-\mathrm{H}$ in heterocyclic ring), $1653.05(\mathrm{C}=\mathrm{O})$, $1388.79(\mathrm{C}-\mathrm{N}), 1089.82(\mathrm{C}-\mathrm{Cl}), 702.11(\mathrm{C}-\mathrm{S})$.

\section{3,3'-(Pyridine-2,6-diyl)bis(5-(2-methoxy arylidene-2-phenyl)thiazolidin-4-one (2j)}

$\mathrm{IR}\left(\mathrm{KBr}, \mathrm{cm}^{-1}\right), v \max =3057.27(\mathrm{Ar}), 3030.27\left(\mathrm{CH}\right.$ in ring), 2933.83( $\left.\mathrm{CH}_{3}\right), 1622.19(\mathrm{C}=\mathrm{O})$, 1394.58(C-N), $1219.05(\mathrm{C}-\mathrm{O}-\mathrm{C}), 640.39$ (C-S). 


\section{3,3'-(Pyridine-2,6-diyl)bis(5-((phenyl) di azenyl)-2-phenyl)thiazolidin-4-one (3a)}

Brown colour powder; $\mathrm{IR}\left(\mathrm{KBr}, \mathrm{cm}^{-1}\right) v \max =3038.76(\mathrm{C}-\mathrm{H}$ in ring), 3019.91( $\left.\mathrm{Ar}), 1595.19(\mathrm{~N}=\mathrm{N})\right)$, 1338.22(C-N), 662.03(C=O). ${ }^{1} \mathrm{H}$ NMR(oppm, DMSO-d6) 5.92(s, 2H, CH), 7.05-7.29(m, 20H, $\mathrm{Ar}-\mathrm{H}), 7.9\left(\mathrm{t}, 1 \mathrm{H}, \mathrm{C}_{4}-\mathrm{Pyridine}\right), 8.2\left(\mathrm{~s}, 2 \mathrm{H}, \mathrm{C}_{5}-\mathrm{H}\right.$ in thiazolidinone $), 8.5\left(\mathrm{C}_{3} \& \mathrm{C}_{5}-\mathrm{H}\right.$ in pyridine $)$.

\section{3,3'-(Pyridine-2,6-diyl)bis(5-((4-nitro phenyl) di azenyl)-2-phenyl)thiazolidin-4-one (3b):}

$\operatorname{IR}\left(\mathrm{KBr}, \mathrm{cm}^{-1}\right) v \max =3047.11(\mathrm{C}-\mathrm{H}$ in ring), 3029.39(Ar), $1665.82(\mathrm{C}=\mathrm{O}), 1602.18(\mathrm{~N}=\mathrm{N})$, $1312.26(\mathrm{C}-\mathrm{N}), 701.03(\mathrm{C}-\mathrm{S})$; ${ }^{1} \mathrm{H}$ NMR( $\left.8 \mathrm{ppm}, \mathrm{DMSO}-\mathrm{d} 6\right)$ 6.1(s, 2H,N-CH-Ar), 7.39(m,18H, $\mathrm{Ar}), 7.9\left(\mathrm{t}, 1 \mathrm{H}, \mathrm{C}_{4}-\mathrm{H}\right.$ in pyvidine), $8.38\left(\mathrm{~d}, 2 \mathrm{H}, \mathrm{C}_{3}, \mathrm{C}_{5}-\mathrm{H}\right.$ in pyridine); ${ }^{13} \mathrm{CNMR}(\mathrm{DMSO}) \delta$ : $171.59,153.35,148.71,135.73,129.8,124.21,119.3,115.27$.

3,3'-(Pyridine-2,6-diyl)bis(5-((4-chloro phenyl) diazenyl)-2-phenyl)thiazolidin-4one $(3 c)$

IR $\left(\mathrm{KBr}, \mathrm{cm}^{-1}\right) v \max =3051.26(\mathrm{C}-\mathrm{H}$-in-ring), 3032.15(Ar), $1656.43(\mathrm{C}=\mathrm{O}), 1599.14(\mathrm{~N}=\mathrm{N})$, 1307.69(C-N), 1091.16(C-Cl), 694.78(C-S); ${ }^{1} \mathrm{H}$ NMR( $\delta$ ppm, DMSO-d6) 5.82(s,2H,N-CH$\mathrm{Ar}), 7.52(\mathrm{~m}, 18 \mathrm{H}, \mathrm{Ar}), 7.85\left(\mathrm{t}, 1 \mathrm{H}, \mathrm{C}_{4}-\mathrm{H}\right.$ in pyridine $), 8.46\left(\mathrm{~d}, 2 \mathrm{H}, \mathrm{C}_{3}, \mathrm{C}_{5}-\mathrm{H}\right.$ in pyridine $) ;{ }^{13} \mathrm{CNMR}$ (DMSO) $\delta: 170.78,152.73,135.2,130.29,122.91,118.2,116.35$.

Table 1. Physical and analytical data of compounds 2a-j \& 3a-c.

\begin{tabular}{|c|c|c|c|c|c|c|}
\hline \multirow[t]{2}{*}{ Compd } & \multirow{2}{*}{$\mathrm{R}$} & \multirow{2}{*}{$\stackrel{\overrightarrow{0}}{\stackrel{\partial}{\nu}} 0^{\circ}$} & \multirow{2}{*}{ ن } & \multirow{2}{*}{ Mol.Formula/Mol.wt } & \multicolumn{2}{|c|}{$\begin{array}{l}\text { Elementalanalysis } \\
\text { (calcd/found) }\end{array}$} \\
\hline & & & & & C & $\mathrm{N}$ \\
\hline $2 \mathbf{a}$ & $4-\mathrm{OH}$ & 67 & 307 & $\mathrm{C}_{37} \mathrm{H}_{27} \mathrm{~N}_{3} \mathrm{O}_{4} \mathrm{~S}_{2} / 641.76$ & $\mathrm{C}(69.25 / 69.28)$, & $(6.55 / 6.54)$ \\
\hline $2 \mathbf{b}$ & $\mathrm{H}$ & 69 & 338 & $\mathrm{C}_{37} \mathrm{H}_{27} \mathrm{~N}_{3} \mathrm{O}_{2} \mathrm{~S}_{2} / 609.76$ & $\mathrm{C}(72.88 / 72.83)$, & $\mathrm{N}(4.46 / 4.45)$ \\
\hline $2 c$ & $4-\mathrm{N}-\left(\mathrm{CH}_{3}\right)_{2}$ & 66 & 293 & $\mathrm{C}_{41} \mathrm{H}_{37} \mathrm{~N}_{5} \mathrm{O}_{2} \mathrm{~S}_{2} / 695.89$ & $C(70.76 / 70.72)$, & $\mathrm{N}(10.06 / 10.02)$ \\
\hline 2d & $3,4,5-\left(\mathrm{OCH}_{3}\right)_{3}$ & 71 & 309 & $\mathrm{C}_{43} \mathrm{H}_{39} \mathrm{~N}_{3} \mathrm{O}_{8} \mathrm{~S}_{2} / 789.92$ & $C(65.38 / 65.42)$ & $\mathrm{N}(5.32 / 5.36)$ \\
\hline $2 e$ & $4-\mathrm{OCH}_{3}$ & 75 & 341 & $\mathrm{C}_{39} \mathrm{H}_{31} \mathrm{~N}_{3} \mathrm{O}_{4} \mathrm{~S}_{2} / 669.81$ & $\mathrm{C}(69.93 / 69.95)$ & $\mathrm{N}(6.27 / 6.28)$ \\
\hline $2 f$ & $2-\mathrm{Cl}$ & 73 & 319 & $\mathrm{C}_{37} \mathrm{H}_{25} \mathrm{Cl}_{2} \mathrm{~N}_{3} \mathrm{O}_{2} \mathrm{~S}_{2} / 678.65$ & $\mathrm{C}(65.48 / 65.48)$ & $\mathrm{N}(6.19 / 6.13)$ \\
\hline $2 \mathrm{~g}$ & $2-\mathrm{Br}$ & 70 & 354 & $\mathrm{C}_{37} \mathrm{H}_{25} \mathrm{Br}_{2} \mathrm{~N}_{3} \mathrm{O}_{2} \mathrm{~S}_{2} / 767.55$ & $\mathrm{C}(57.90 / 57.93)$ & $\mathrm{N}(5.47 / 5.46)$ \\
\hline $2 h$ & $4-\mathrm{NO}_{2}$ & 74 & 301 & $\mathrm{C}_{37} \mathrm{H}_{25} \mathrm{~N}_{5} \mathrm{O}_{6} \mathrm{~S}_{2} / 699.75$ & $\mathrm{C}(63.51 / 63.5)$ & $\mathrm{N}(10.01 / 10.07)$ \\
\hline $2 \mathbf{i}$ & $4-\mathrm{Cl}$ & 69 & 317 & $\mathrm{C}_{37} \mathrm{H}_{25} \mathrm{Cl}_{2} \mathrm{~N}_{3} \mathrm{O}_{2} \mathrm{~S}_{2} / 678.65$ & $C(65.48 / 65.45)$ & $\mathrm{N}(6.19 / 6.17)$ \\
\hline $2 \mathbf{j}$ & $2-\mathrm{OCH}_{3}$ & 71 & 299 & $\mathrm{C}_{39} \mathrm{H}_{31} \mathrm{~N}_{3} \mathrm{O}_{4} \mathrm{~S}_{2} / 669.81$ & $\mathrm{C}(69.93 / 69.91)$, & $\mathrm{N}(6.27 / 6.23)$ \\
\hline $3^{\mathrm{a}}$ & $\mathrm{H}$ & 79 & 310 & $\mathrm{C}_{35} \mathrm{H}_{27} \mathrm{~N}_{7} \mathrm{O}_{2} \mathrm{~S}_{2} / 641.76$ & $C(65.50 / 65.53)$ & $\mathrm{N}(15.28 / 15.27)$ \\
\hline $\mathbf{3 b}$ & $4-\mathrm{NO}_{2}$ & 81 & 327 & $\mathrm{C}_{35} \mathrm{H}_{25} \mathrm{~N}_{9} \mathrm{O}_{6} \mathrm{~S}_{2} / 731.76$ & $C(57.45 / 57.48)$ & $\mathrm{N}(17.23 / 17.26)$ \\
\hline $3 \mathbf{c}$ & $4-\mathrm{Cl}$ & 84 & 315 & $\mathrm{C}_{35} \mathrm{H}_{25} \mathrm{Cl}_{2} \mathrm{~N}_{7} \mathrm{O}_{2} \mathrm{~S}_{2} / 710.65$ & $\mathrm{C}(59.15 / 59.14)$ & $\mathrm{N}(13.80 / 13.78)$ \\
\hline
\end{tabular}

\section{Results and Discussion}

The key intermediate 1 was prepared by a one pot three component condensation reaction involving 2, 6-di amino pyridine, benzaldehyde, and mercaptoacid in the presence of a $\gamma$-ferrite as a catalyst (18). The synthetic route of the compounds is outlined in Scheme-l. This Thiazolidinone(1) reacted with various araldehydes afforded to bis thiazolidinones and 
their 5-arylidene derivatives(2a-j) through knoevenagel reaction. In another way Diazotization of compounds with various aromatic amines yielded azo compounds (3a-c). The newly synthesized compounds were characterized by FT-IR, NMR $\left({ }^{1} \mathrm{H},{ }^{13} \mathrm{C}\right)$ spectral data.

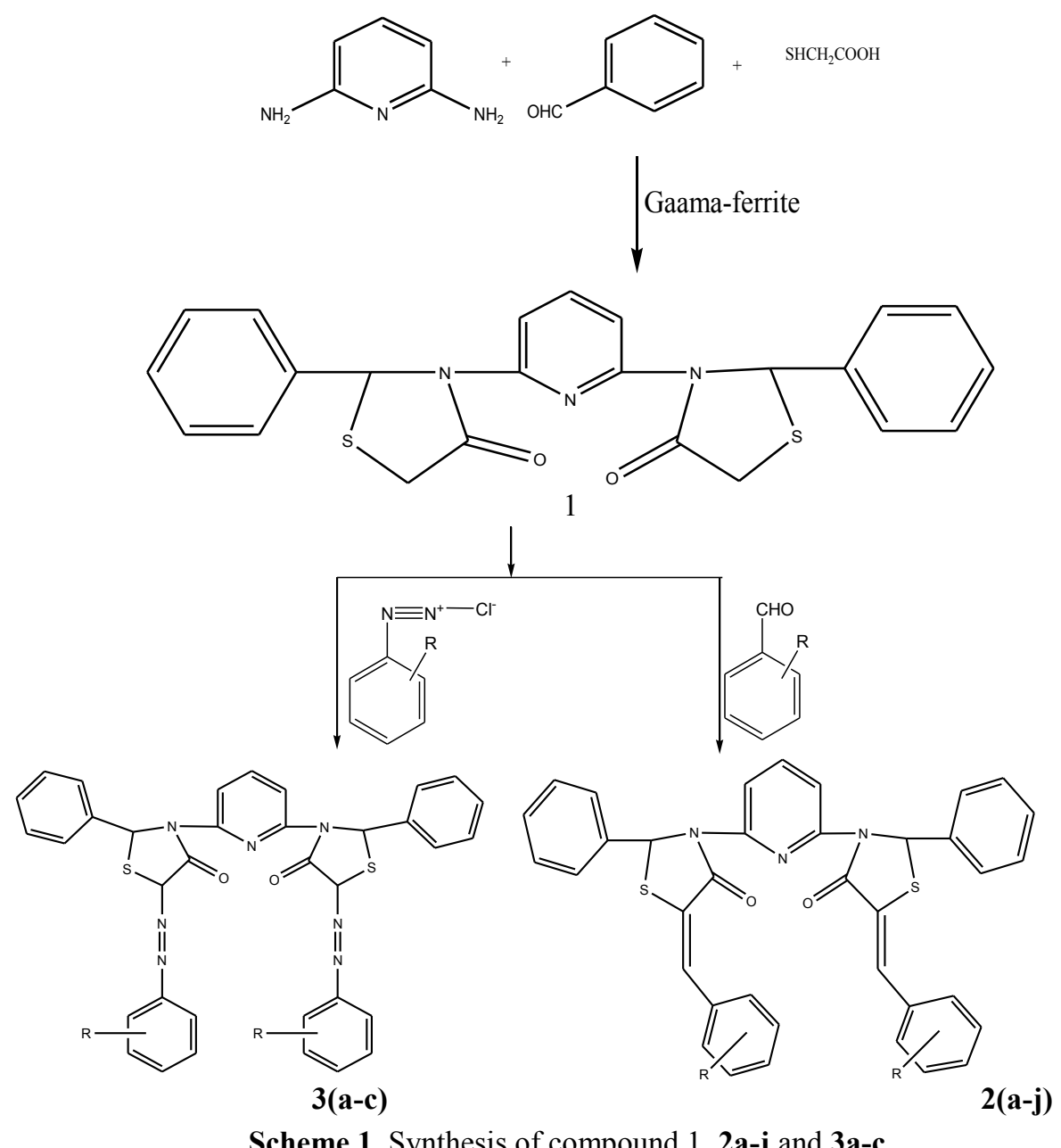

Scheme 1. Synthesis of compound 1, 2a-j and 3a-c.

\section{Anti Microbial Evaluation}

Thirteen of the newly synthesized compounds were evaluated for their in vitro antibacterial activity against $B$. subtilis, $B$. thuringiensis, E. coli and P. aeruginosa, as well as antifungal activity against $B$. fabae, $F$. oxysporam and $T$. viridae organisms. Agar diffusion method was used for the determination of the preliminary anti bacterial and antifungal activities. Streptomycin, Chloramphenicol and Treflucan were used as reference drugs. The results depicted in Table 2 revealed that most of tested compounds displayed variable inhibitory effects on the growth of the tested organisms. Regarding the activity of the thiazolidinone derivatives against bacteria, the results revealed that compounds $\mathbf{2 f - 2 i}, \mathbf{3 b}$, and $\mathbf{3 c}$ exhibited broad spectrum of antibacterial profile against the tested organisms. 
Table 2. Antimicrobial activity data of compounds $\mathbf{2 a - j} \& \mathbf{3 a - c}(\mathrm{MIC}, \mu \mathrm{g} / \mathrm{mL})$.

\begin{tabular}{|c|c|c|c|c|c|c|c|}
\hline Compd No & 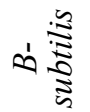 & $\begin{array}{c}B . \\
\text { thuringiensis }\end{array}$ & E-Coli & $\begin{array}{c}P . \\
\text { aeruginosa }\end{array}$ & $\begin{array}{c}\text { B. } \\
\text { fabae }\end{array}$ & $\begin{array}{c}F . \\
\text { Oxysporam }\end{array}$ & $\begin{array}{l}\text { Trichoder } \\
\text { maviridae }\end{array}$ \\
\hline $2 \mathbf{a}$ & 100 & 200 & 100 & 100 & 50 & 50 & 100 \\
\hline $2 \mathbf{b}$ & 100 & 50 & 50 & 100 & 100 & 50 & 200 \\
\hline $2 c$ & 200 & 100 & 200 & 50 & 50 & 100 & 50 \\
\hline 2d & 50 & 100 & 100 & 100 & 25 & 12.5 & 12.5 \\
\hline $2 e$ & 50 & 100 & 50 & 50 & 6.25 & 12.5 & 6.25 \\
\hline $2 f$ & 25 & 50 & 50 & 100 & 50 & 25 & 25 \\
\hline $2 \mathrm{~g}$ & 25 & 50 & 50 & 12.5 & 50 & 25 & 25 \\
\hline $2 \mathrm{~h}$ & 12.5 & 25 & 50 & 50 & 25 & 12.5 & 25 \\
\hline $2 \mathbf{i}$ & 6.25 & 12.5 & 25 & 25 & 25 & 50 & 50 \\
\hline $2 \mathbf{j}$ & 100 & 50 & 100 & 100 & 12.5 & 6.25 & 25 \\
\hline $3 \mathbf{a}$ & 200 & 100 & 50 & 100 & 100 & 50 & 100 \\
\hline $3 \mathbf{b}$ & 12.5 & 25 & 25 & 12.5 & 12.5 & 25 & 25 \\
\hline $3 c$ & 12.5 & 25 & 25 & 50 & 12.5 & 6.25 & 12.5 \\
\hline Streptomycin & 3.125 & 6.25 & 6.25 & 6.25 & - & - & - \\
\hline Chloramphenicol & 6.25 & 6.25 & 6.25 & 6.25 & - & - & - \\
\hline Treflucan & - & - & - & - & 3.125 & 3.125 & 3.125 \\
\hline
\end{tabular}

Regarding the activity of thiazolidinones and their 5- arylidenes and their 5- azo derivatives incorporating pyridine moiety against antifungal strains the results revealed that compounds $\mathbf{2 e}, \mathbf{2} \mathbf{j}, \mathbf{3 b}$, and $\mathbf{3} \mathbf{c}$ revealed strong growth inhibitory against the tested fungi.

\section{Conclusion}

In conclusion, the objective of the present study was to synthesize and investigate the antimicrobial activities of some new heterocyclic derivatives by the use of $\gamma$-ferrite leads serving as potent antimicrobial agents. Our aim has been verified by the synthesis of two different groups of structure hybrids comprising basically the pyridine moiety attached to either arylidene substituted thiazolidinones (or) azo substituted thiazolidinones. The obtained results clearly revealed that some of the newly synthesized compounds exhibited better anti microbial activity.

\section{Acknowledgment}

The authors are grateful to The Head, Department of Chemistry Dr. H. S. Gour University Sagar for providing necessary facilities to carry out this work. We sincerely express our gratitude to Head, Biotechnology laboratory, Department of Botany, Dr. H. S. Gour University Sagar for providing antimicrobial data. We are grateful to The Director, SAIF, Panjab University for providing spectral data.

\section{References}

1 Horton D A, Bourner G T and Smythe M L, Chem Rev., 2003, 103, 893.

2 Thompson L A and Ellman J A, Chem Rev., 1996, 96, 555.

3 Vigorita M G, Ottana R, Monforte F, Maccari R, Trovato A, Monforte M T and Traviano M F, Bioorg Med Chem Lett., 2001, 11, 2791.

$4 \quad$ Singh S P, Parmar S S, Raman K and Stenberg V I, Chem Rev., 1981, 81, 175.

5 Sobin B A, J Am Chem Soc., 1952, 74, 2947.

6 Kucukguzel G, Kocatepe A, De Clercq E, Sahin F and Gulluce M, Eur.J Med Chem., 2006, 41, 353.

7 Babaoglu K, Page M A, Jones V C, Mc.Neil M R, Dong C, Naismith J H and Lee R E, Bioorg Med Chem Lett., 2003, 13, 3227. 
Look G C, Schullek J R, Holmes C P, Chinn J P, Gorden E M and Gallop M A, Bioorg Med Chem Lett., 1996, 6, 707.

9 Srivastava S K, Yadav R and Srivastava S D, J Indian Chem Soc., 2004, 81, 342.

10 Previtera T, Vigorita M G, Bisila M, Orsini F, Benetolla F and Bombieri G, Eur $J$ Med Chem., 1994, 29, 317.

11 Barreca M L, Chimirri A, Luca L D, Monforte A M, Monforte P, Rao A, Zappala M, Balzarini J, Clercq E De, Pannecouque C and Witvrouw M, Bioorg Med Chem Lett., 2001, 11, 1793.

12 Asati K C, Srivastava S K and Srivastava S D, Indian J Chem., 2006, 45B, 526.

13 Srivastava S K, Srivastava S and Srivastava S D, Indian J Chem., 2002, 41B, 1937.

14 Kato T, Ozaki T and Ohi N Tetrahedron Asymmetry, 1993, 10, 3963.

15 Bhatt J J, Shah R B, Shah P H, Trivedi B P, Undavia K N and Desai C N, Indian J Chem., 1994, 33B, 189.

16 Rawal R K, Prabhakar Y S, Katti S B and De Clercq E, Bioorg Med Chem 2005, 13, 6771.

17 Andres C J, Bronsen J J, Andrea S V D, DeshPande M S, Falk P J, Grant young K A, Harte W E, Ho H T, Misco P F, Robertson J G, Stock D, Sun Y and Walsh A W, Bioorg Med Chem Lett., 2000, 10, 715.

18 Sadashiva C T, Narendra Sarath Chandra J N, Kavitha C V, Thimmegowda A, Subhash M N and Rangappa Kanchugaragoppal S, Eur J Med Chem., 2009, 44, 4848. 


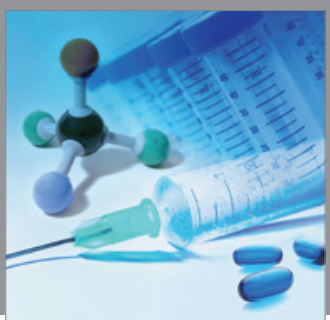

International Journal of

Medicinal Chemistry

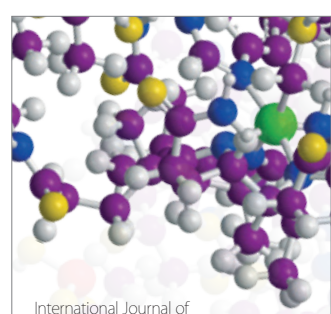

Carbohydrate Chemistry

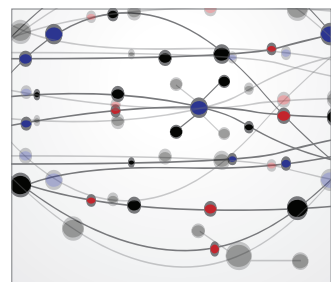

The Scientific World Journal
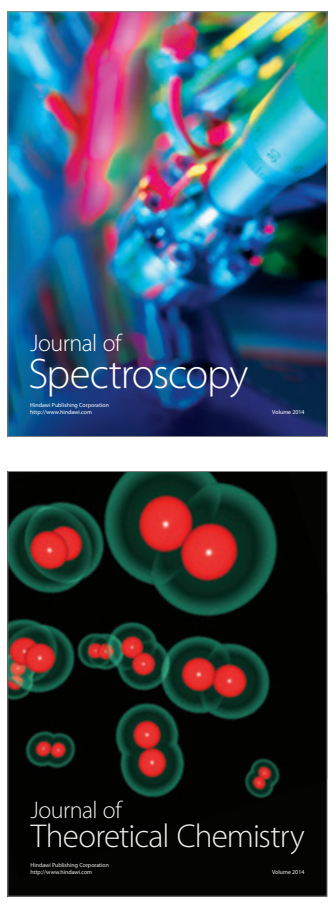
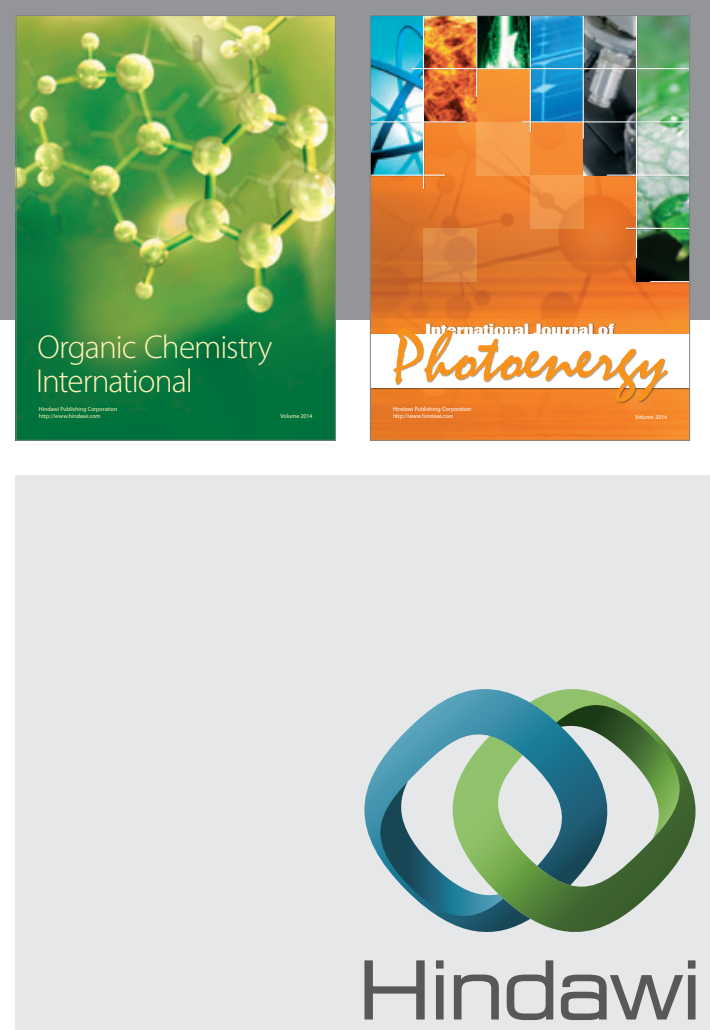

Submit your manuscripts at

http://www.hindawi.com
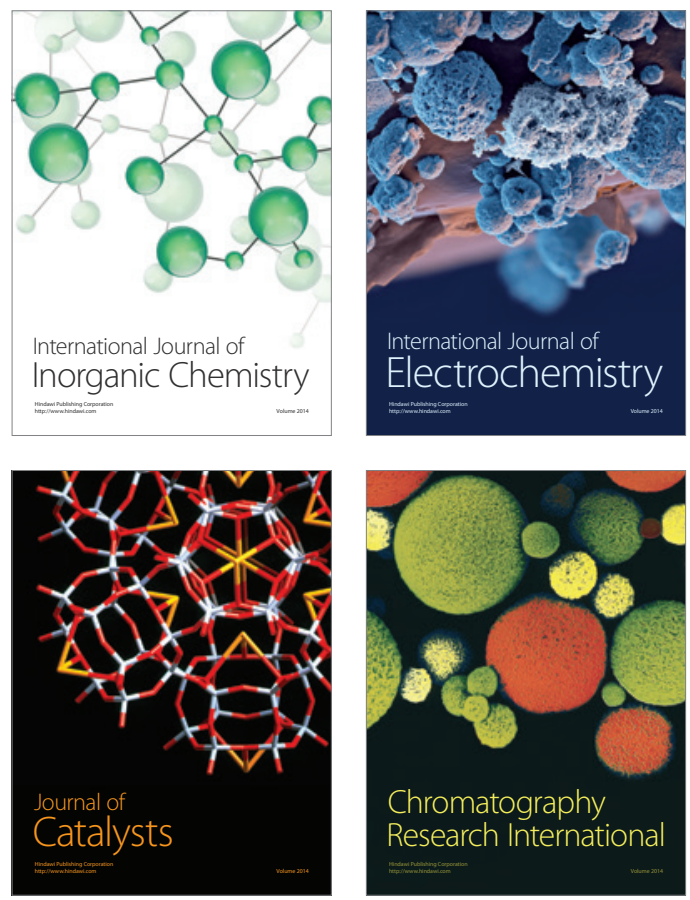
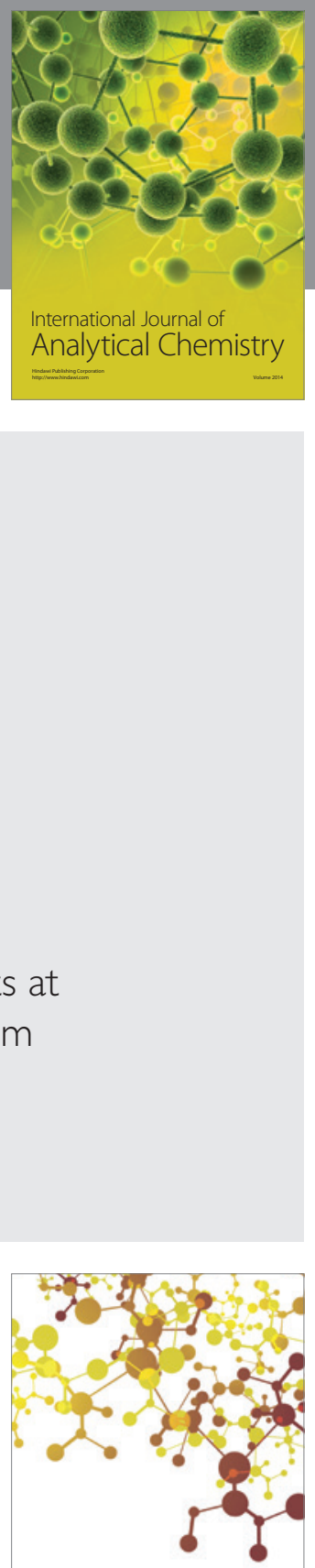

Journal of

Applied Chemistry
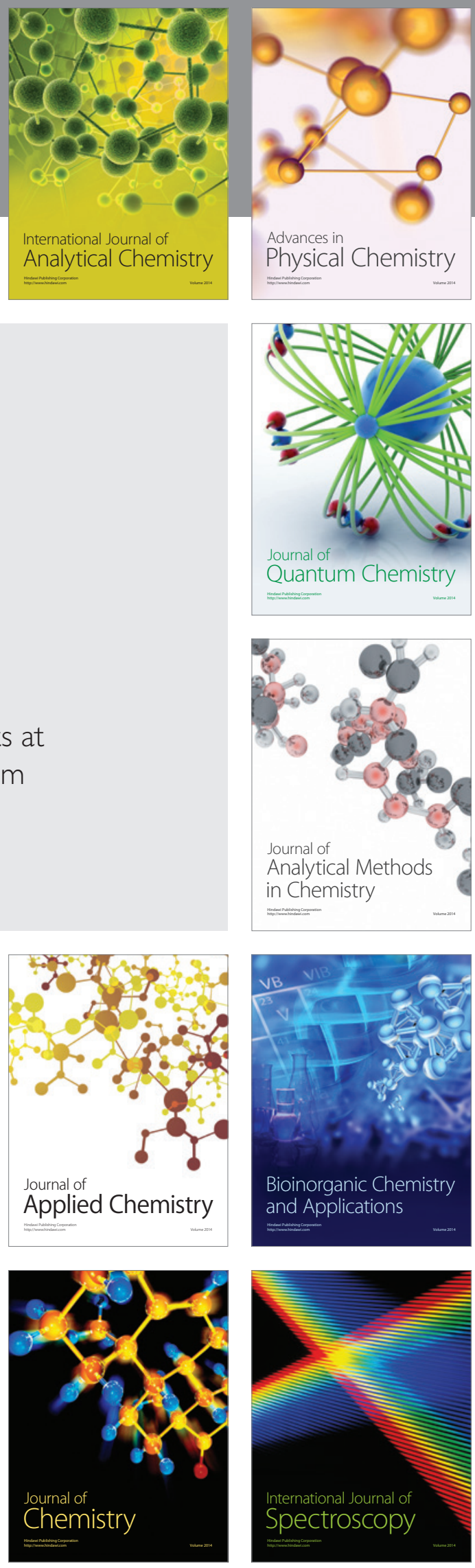